\title{
Desde las prácticas ecológicas y familiares hacia una economía social y solidaria. La experiencia de la Asociación de Productores Ecológicos del Valle del Chillón
}

\section{From ecological and family practices to a social and solidarity economy. The experience of the Association of Ecological Producers of the Chillón Valley}

\author{
Fabiola Pereda Anco \\ Zoila Diaz León \\ Alejandro Zamudio Ariza \\ https://orcid.org/0000-0001-8657-0052 https://orcid.org/0000-0001-7348-7743 https://orcid.org/0000-0003-4554-1727 \\ fabiola.pereda1@unmsm.edu.pe \\ zoila.diaz1@unmsm.edu.pe \\ andres.zamudio@unmsm.edu.pe \\ Universidad Nacional Mayor de San Marcos
}

\begin{abstract}
RESUMEN
En la presente investigación se busca saber si las dinámicas de organización y producción de la Asociación de Productores Ecológicos del Valle del Chillón (APEVCH), ubicada en la cuenca baja del valle del Chillón, representan un sistema económico de tipo social y solidario. Para ello, se han realizado entrevistas principalmente a los miembros de la junta directiva, estructuradas sobre cuatro ejes. El primero es sobre la recuperación de memoria, para entender qué gestó la conformación de la APEVCH; el segundo está orientado a describir las relaciones internas para analizar la forma en la que se organizan; el tercero trata sobre las relaciones externas, que está abocado a indagar acerca de las redes con actores gubernamentales, actores locales y su despliegue económico (mercados); el cuarto, finalmente, gira en tonro al eje de las perspectivas a futuro. Todo ello para desentrañar las dinámicas que se practican en la APEVCH. De la experiencia, se pudo apreciar que se practica un modo de producción bajo nociones ecológicas, que generan prácticas orientadas principalmente a la satisfacción de las necesidades básicas de los agricultores y sus familias, mientras que el excedente de producción es orientado al comercio. A su vez, ello genera relaciones que se presentan dentro de la asociación, basadas principalmente en la solidaridad.
\end{abstract}

Palabras clave: Agricultura periurbana; economía social y solidaria; producción ecológica.

\begin{abstract}
This research seeks to answer whether the organization and production dynamics of the "Association of Ecological Producers of the Chillón Valley" (APEVCH), located in the lower Chillón valley basin, represent a social and solidarity-based economic system. To accomplish this, interviews have been carried out mainly with the members of the board of directors structured on four axes. The first one is about the recovery of memory to understand what led to the formation of the APEVCH; the second one is oriented to describe the internal relations to analyze the way they are organized; the third one is about the external relations, which is devoted to investigating the networks with government actors, local actors and their economic deployment (markets); and the fourth axis refers to the future perspectives. All this is to unravel the dynamics practiced in APEVCH. The experience showed that a mode of production is practiced under ecological notions, which generate practices oriented mainly to the satisfaction of the basic needs of farmers and their families, while the surplus production is oriented to trade. In turn, this generates relationships that are presented within the association and are based mainly on solidarity.
\end{abstract}

Keywords: Periurban agriculture; social and solidarity economy; ecological production.

(C) Los autores. Este artículo es publicado por ISHRA, Revista del Instituto Seminario de Historia Rural Andina de la Facultad de Ciencias Sociales de la Universidad Nacional Mayor de San Marcos. Este es un artículo de acceso abierto, distribuido bajo los términos de la licencia Creative Commons Atribución 4.0 Internacional (CC BY 4.0) [https://creativecommons.org/licenses/by/4.0/deed.es] que permite el uso, distribución y reproducción en cualquier medio, siempre que la obra original sea debidamente citada de su fuente original. 


\section{Introducción}

La Asociación de Productores Ecológicos del Valle del Chillón (en adelante, APEVCH), se caracteriza por estar conformada por agricultores que practican la pequeña agricultura de tipo familiar y periurbana. La definición de agricultura familiar es, según la Estrategia Nacional de Agricultura Familiar 2015-2021 (en adelante, ENAF) que adoptó desde la Comisión Multisectorial por el Año Internacional de la Agricultura Familiar - CAIAF (2015), un modo de vida y producción que practican hombres y mujeres de un mismo núcleo familiar, en un territorio en el que están a cargo de sistemas productivos desarrollados en la unidad productiva familiar. Estas a su vez están vinculadas y combinan funciones económicas, ambientales, reproductivas, sociales y culturales. En otras palabras, la fuerza de trabajo predominante es familiar, lo cual se convierte en una de las múltiples fuentes de ingresos; tiene un acceso limitado a recursos de tierra, agua y capital; y forma redes de reciprocidad donde el rol de la mujer es relevante en la actividad productiva y reproductiva.

Siguiendo las pautas de los estudios de la FAO (2007), las tipologías de agricultura familiar que considera la ENAF son tres: subsistencia, intermedia y consolidada. Los integrantes de APEVCH se encuentran ubicados dentro de la agricultura familiar intermedia, ya que, según su definición, presenta una mayor dependencia de su actividad como la venta y autoconsumo, pero no llegan a generar un excedente suficiente para expandir su fuerza de trabajo y adquirir nueva tecnología para desarrollar la agricultura.

Ahora, según la ubicación, APEVCH pertenece a un tipo de agricultura periurbana, la cual, tomando el estudio realizado por el Comité de Agricultura de la FAO (1999), se refiere a unidades agrícolas cercanas a una ciudad que explotan intensivamente granjas comerciales o semicomerciales para cultivar hortalizas y otros productos hortícolas, criar aves u otros animales y producir leche y huevos.

Si bien se podría decir que la agricultura familiar en el Perú encuentra sus orígenes en procesos históricos como la reforma agraria, cuya ley se promulgó en el año 1969, no es hasta la década de los noventa que es considerarada como una forma de manejo de unidades productivas (o parcelas). La costa peruana, para el año 2018, es la tercera región con mayor despliegue de actividades agrícolas de tipo familiar.

La revisión de la literatura sobre estudios en el valle del Chillón muestra investigaciones dedicadas a poner en evidencia los efectos de la urbanización, que trae conflictos por el cambio de uso de los suelos y el manejo de los recursos hídricos; o el uso de agroquímicos para el aumento de la productividad y sus impactos en el entorno ecosistémico, desde una perspectiva ambiental y económica. También muestra otras apreciaciones derivadas del análisis de experiencias desenvueltas a partir de la implementación de proyectos.

Respecto a los conflictos generados por el cambio del uso de los suelos, las inmobiliarias formales e informales no respetan las zonas agrícolas, canales de riego, ni la zonificación o planificación urbana, operando mediante la compra de parcelas de manera individual y lotizando de forma precaria los terrenos. Además, algunas normas, como la ordenanza 548, aprobó el ingreso sin restricciones de las inmobiliarias al valle, lo que permitió la consolidación de los espacios urbanos, pasando por alto su depredación y destrucción (Cáceres, 2013).

El uso de los recursos hídricos en el valle del Chillón provoca también conflictos, debido a las tensiones con otras actividades como la crianza de ganado porcino y plantas de fundición clandestina, que terminan convirtiéndose en fuente principal de contaminación a lo largo de la 
cuenca baja del río Chillón (Aliaga, 2010), así como conflictos entre los usuarios de riego y los pobladores aledaños, debido a los residuos sólidos que vierten en el río a causa de la ausencia de servicios básicos de saneamiento (Mondragón, 2019).

Otro asunto de atención es el uso de agroquímicos. La información disponible permite apreciar que, para el año 2015, el valle del Chillón presentaba un uso indiscriminado de plaguicidas debido al escaso conocimiento sobre la forma de uso y los efectos negativos de los mismos para la salud (Díaz, 2019). Asimismo, los agricultores carecen de equipos de aplicación y protección, y de desecho y reciclaje de los envases, afectando al consumidor y al agricultor (Marañón, 2015).

Según un análisis sobre la sostenibilidad de las fincas de brócoli, realizado en el año 2017, se mantiene el uso de pesticidas, principalmente, por parte de agricultores no organizados, lo cual no permite su sostenibilidad en la dimensión ambiental (Andrade, 2017); sin embargo, en la dimensión económica, «el riesgo económico en general está bien manejado debido a la diversificación de la producción y tener más de cinco canales de comercialización» (Andrade, 2017, p. 138).

Finalmente, se encontró un informe elaborado a partir de un proyecto realizado por FOVIDA y cuya intervención se desarrolló en el año 2010, en el cual se detectaron ciertas falencias por parte de los agricultores del valle del Chillón, entre las cuales se incluían un manejo productivo que no les permitía hallar un equilibrio en cuanto a sostenibilidad se refiere. Una propuesta era constituir una sola organización de las y los productores, que les permitiría el avance; sin embargo, a ello se sumaba la falta de diálogo para establecer acuerdos colectivos. Por ello, para lograr un mejor desempeño de las actividades agrícolas, se proponía de manera complementaria la planificación de siembra y cosecha, la adquisición de semillas e insumos, labores de post-cosecha o transporte a la empresa, y mejora de capacidades de los diversos actores que se veían implicados en el proceso de inserción al mercado (Cuentas et al., 2010).

Estos problemas están presentes no solo en el valle del Chillón sino en todos los espacios en los que se desenvuelven actividades de agricultura periurbana. Estos problemas motivaron las investigaciones revisadas, que analizaron problemas del valle del Chillón desde distintas perspectivas disciplinarias, en especial, la geografía y la ingeniería ambiental.

Consideramos que una perspectiva desde las ciencias sociales podría ayudar a tomar en cuenta aspectos que se están ignorando en los estudios sobre los problemas que se presentan en el valle del Chillón. Esta perspectiva integra la perspectiva económica y cultural, en las cuales se toma principalmente el desarrollo de los pobladores locales, basada en sus propias nociones de desarrollo, para lo cual es indispensable asumir como objeto de estudio las relaciones que se generan y establecen dentro de la organización.

Nuestra pesquisa busca responder la interrogante: ¿La APEVCH, a través de sus prácticas de producción ecológica y familiar, generan relaciones que constituyen una economía social y solidaria? ${ }^{1}$

La metodología del trabajo prioriza un abordaje de tipo cualitativo, desenvuelto a través de entrevistas a los integrantes de la APEVCH; sin embargo, debido a las limitaciones generadas por la coyuntura de crisis sanitaria desde comienzos del 2020, estas se realizaron por videollamada. Los entrevistados fueron, principalmente, cinco miembros de la actual junta directiva.

1 Es decir, una economía que produce sociedad y no sólo utilidades económicas, genera valores de uso para satisfacer las necesidades de los productores, no esta orientada a la acumulación sin límites de capital, y recupera la unión entre producción y reproducción. Se debe tomar en cuenta que la producción de la sociedad está determinada por la noción del buen vivir, la cual mantiene como base el régimen de la solidaridad (Coraggio, 2011). 
También fueron analizados documentos especializados sobre agricultura familiar y periurbana en el caso del valle del Chillón.

Tomaremos como indicadores que la organización genera relaciones basadas en la solidaridad, reciprocidad y horizontalidad dentro de la asociación, de forma que rompe con las relaciones de acumulación de capital como eje central, reemplazándolo con el establecimiento de lazos en los que predomina el respeto y cuidado de la vida humana y la naturaleza.

El trabajo desarrolla como primer punto la memoria histórica de la APEVCH, para poder establecer los sucesos que generaron su conformación; luego, el segundo describe sus relaciones internas, las cuales sirven para entender cómo se organiza y qué es lo que determina el tipo de vínculos que establece; después, el tercero examina las relaciones externas que posee, para indagar en sus lazos con agentes, tales como las municipalidades, las ONG y los dirigentes de las zonas pobladas aledañas. El cuarto punto presenta las perspectivas a futuro de la APEVCH. Por último, el trabajo cierra con las conclusiones, de acuerdo a la experiencia y al acercamiento desenvuelto desde la pesquisa realizada.

\section{Memoria histórica}

\subsection{Antecedentes}

Situados bajo la jurisdicción de las municipalidades del distrito de Carabayllo, de la provincia de Lima, y del distrito de Santa Rosa de Quives, de la provincia de Canta, encontramos a diversos agricultores que desempeñan sus actividades productivas en el valle del Chillón. Ellas y ellos se encuentran distribuidos en parcelas, que son trabajadas de manera individual o familiar, donde realizan el cultivo de hortalizas, que es una de las principales actividades económicas que los sustenta. Poseen juntas y comités de riego como una forma de llevar adelante un manejo adecuado del agua del río Chillón. Esta es una de las formas de organización más visibles en el valle.

Con el acelerado proceso de urbanización metropolitano que se desarrolló en la ciudad de Lima, se fueron viendo afectadas las áreas destinadas a la agricultura en la cuenca baja del valle del Chillón, debido al tráfico de tierras para producir cemento.

Las denuncias efectuadas durante años, por parte de agricultores y/o personas que habitan el valle, fueron recibidas por las autoridades de la Municipalidad Distrital de Carabayllo y la Municipalidad Distrital de Santa Rosa de Quives, sin hacer nada al respecto. Y sumado a ello, se evidenció el uso indiscriminado de agroquímicos que se empleaban para los cultivos, dañando la tierra, generando toxicidad en los alimentos, enfermedades en quienes los empleaban y a su vez en las personas que los consumían, así como consecuencias desastrosas para el ambiente.

\subsection{De lo singular a lo plural}

Frente a los problemas expuestos, se evidenció que no existía en el valle una organización de productores que se encargara de la administración y gestión de la sostenibilidad de la producción, además de contar con muy poca o nula intervención de proyectos de desarrollo, en al menos los últimos diez años.

Un pequeño grupo, ya instaurado, fue casa por casa a hacer extensiva la invitación para poder sumarse a esta iniciativa que buscaba responder a las problemáticas instituidas en el va1le. Es así como la señora Yanina Loayza lo declaró: «siendo esposa de un agricultor y sin saber absolutamente nada de lo que era producir hortalizas y trabajar las tierras de manera ecológica, recibí la invitación para poder ser partícipe de esta asociación que de manera novedosa propuso 
producir ecológicamente, que era algo a lo que muchos no estaban acostumbrados y que me llamó la atención» (Y. Loayza, entrevista, 17 de septiembre de 2020).

Es así que uno a uno se fue sumando a esta iniciativa, siendo parte de las reuniones en donde se abordaban los propósitos que buscaban alcanzar y brindar capacitaciones a los y las agricultoras del valle con el fin de brindar, de forma equitativa, las mismas oportunidades de producir de manera alternativa y sostenida.

Durante varios años estuvieron funcionando de manera improvisada, pero al llegar a concretar un mayor número de personas, formaron la Asociación de Productores Ecológicos del Valle Chillón - APEVCH, la cual se instauró en febrero del 2016 de manera formal, con 30 asociados que procuraban hacer visibles los problemas que aquejan al valle y a los diversos agricultores; a la par, ellos y ellas buscan promover una agricultura amigable con el ambiente y ecológica en sus propósitos.

\subsection{Propósitos de APEVCH}

La APEVCH se planteó dos propósitos fundamentales, de acuerdo a lo señalado por Luis Gomero:

Primer propósito: Visibilizar la problemática del valle Chillón a los municipios locales y a la Municipalidad Metropolitana de Lima, debido a que este es uno de los últimos valles con el que cuenta Lima que aún posee tierras agrícolas, los otros son Lurín y Huachipa.

Segundo propósito: No seguir produciendo en la forma en que todos los agricultores lo hacen, debido a que el uso indiscriminado de agroquímicos es alarmante y no cumplen con ninguno de los protocolos indicados por SENASA, por lo tanto, los productos que salen al mercado posiblemente terminen con una cantidad significativa de residuos tóxicos que pongan en peligro la salud de los consumidores de Lima metropolitana, que es su principal mercado. (L. Gomero, entrevista, 13 de agosto de 2020)

\section{Relaciones internas}

La APECH cuenta con 30 socios distribuidos en los distritos de Carabayllo (provincia de Lima) y Santa Rosa de Quives (provincia de Canta), de los cuales solo 10 están activos actualmente.

\subsection{Organización}

La asociación está inscrita en registros públicos y se rige bajo un estatuto aprobado, en el cual se suscribe una junta directiva que les permite organizarse. Esta junta directiva se elige por elecciones internas cada dos años. Sus miembros, con el fin de lograr cada vez una mayor participación, pueden ser reelegidos una sola vez, cumpliéndose así que cada persona ejerza su cargo dentro de un periodo máximo de cuatro años. Según lo mencionado en la entrevista con el señor Felipe, el único requisito para presentarse a cualquier cargo es ser socio. Otro que cabe resaltar es que la elección de una nueva junta ha tenido que ser postergada por la coyuntura actual, por lo que, de manera excepcional, se ha tenido que mantener la misma junta.

Por otro lado, para poder definir si un miembro se encuentra activo o inactivo, como lo menciona la señora Yanina, se toma en cuenta dos cuestiones. La primera está relacionada a la producción ecológica, la cual también es requisito para ser socio de APEVCH. La segunda cuestión está más relacionada con mantener una participación regular. Este punto se refiere a las actividades que, según lo mencionado en las entrevistas, se dan de forma semanal o quincenal. En 
sus palabras: «Un requisito es cultivar y demostrar que estamos haciendo productos ecológicos y naturales, otro requisito era asistir a las reuniones y las charlas que se dan en la asociación» (Y. Loayza, entrevista, 17 de septiembre de 2020). Este tema también se ha tenido que replantear por la coyuntura, ya que algunos representantes tienen limitaciones de hábitos y de tecnología para acceder a las reuniones que se dan mediante plataformas virtuales.

Para indagar sobre el cumplimiento de los indicadores mencionados en la metodología, es decir, la solidaridad, reciprocidad y horizontalidad dentro de la asociación, se estructuró una entrevista en la que se pide que se describan las relaciones de los socios, por medio de las experiencias de los miembros de la junta directiva.

Debido a que esta organización está conformada por grupos de familias que practican la agricultura a pequeña escala, la información sobre la forma en la que se desempeñan las actividades productivas no es muy precisa por los diferentes manejos sobre las distintas unidades productivas. Sin embargo, lo que se puede rescatar de las entrevistas es que lo que se trata de transmitir desde la Asociación, hacia las y los representantes y agricultores que realizan sus actividades en cada grupo, son los principios de solidaridad que, dentro de la organización y de los grupos que lo conforman, se entiende de dos formas: uno hacia adentro y otro hacia afuera.

Cuando mencionamos los principios de solidaridad hacia adentro nos referimos a que las personas que forman parte de la organización presentan una relación horizontal en la cual todos trabajan con el fin de mejorar la situación de las y los mismos agricultores, lo que es descrito por la vicepresidenta: «Se trabaja para todos, de una forma igual o equitativa para todos, pensando en el bienestar de todos, y aunque haya una junta directiva, hay mucho respeto entre todos y con cada miembro» (G. Igreda, entrevista, 05 de setiembre de 2020). De esta manera, se rompe con la idea de competencia para reemplazarla por dinámicas de comunidad, tomando lo mencionado en las entrevistas, todas las labores que realiza la asociación están orientadas a que los grupos de agricultores puedan mantener las prácticas de producción ecológica en el tiempo, y de esta forma se dé un desarrollo de la actividad de forma sostenible.

Cuando mencionamos los principios de solidaridad hacia afuera, según lo señalado por el presidente de la Junta Directiva de APEVCH, se refieren a establecer una relación en la cual se vean beneficiados tanto las y los productores como las y los consumidores. Esta relación se daría a cabalidad mediante el establecimiento de un "precio justo". Esto es, en palabras del Ing. Luis: "cuando estamos trabajando en establecer las cadenas cortas de comercialización, es para facilitar el encuentro entre el productor y el consumidor, y a partir de eso encontrar un precio justo para ambos" (L. Gomero, entrevista, 13 de agosto de 2020). El precio justo estaría relacionado básicamente con la calidad de un producto completamente natural, tal es así que, para establecer mayor confianza con los consumidores, se les invita a un recorrido para que puedan observar los procesos por los cuales se produce el bien que se les está ofreciendo.

\subsection{Cuestiones de género y edad}

Con respecto a la cuestión de género, se observa que en la junta directiva hay una considerable participación femenina. Así también, entre los representantes (asociados) y los miembros de la junta directiva de APEVCH, hay más mujeres que hombres ejerciendo labores; es decir, hay más mujeres activas dentro de la asociación, sea capacitando a los nuevos grupos, o desarrollando actividades de producción agrícola. Cabe resaltar que, según el Ing. Luis, hay mujeres que manejan pequeñas extensiones de tierra, en donde ellas mismas se encargan de la producción, independientemente de otras mujeres que desarrollan esta actividades en sus grupos familiares. 
Como se menciona en la primera parte de esta sección, las actividades productivas las maneja cada socio sobre su unidad productiva, y básicamente éstos son grupos familiares. Por ello, el tema de género en los procesos de producción no es homogéneo; sin embargo, según lo mencionado durante las distintas entrevistas, se puede resaltar una tendencia hacia la división del trabajo entre ambos grupos, en donde las actividades de la producción agraria son generalmente realizadas por mujeres, como comenta el señor Luis: «aproximadamente un $70 \%$ de la fuerza laboral en las actividades en el campo son realizadas por las mujeres, debido a que los hombres ya no quieren trabajar en el campo y prefieren las actividades de transporte» (L. Gomero, entrevista, 13 de Agosto de 2020). En cuanto a los huertos que tienen la oportunidad de contratar jornaleros, es mucho más heterogéneo, ya que, como fue mencionado en todas las entrevistas, hay algunas unidades en las que se prefiere contratar a jornaleros varones, porque los relacionan con el uso de fuerza física, mientras que para otros huertos les es indiferente el tema del género y contratan a quienes quieran trabajar en el campo.

Con respecto al tema generacional, al ser grupos familiares, las actividades en el campo son realizadas por adultos y jóvenes de la misma manera. No hay una división, y la participación no está determinada por un tema netamente de la elección de desempeñar esas actividades o no, sino del desarrollo familiar.

\section{Relaciones externas}

A raíz de los problemas que surgieron en el valle, las y los productores tuvieron un primer acercamiento con las municipalidades de Carabayllo y Santa Rosa de Quives, con la finalidad de solucionar lo que les aqueja.

A partir de ello, con el nacimiento de la APEVCH, han tenido un acercamiento más recurrente, únicamente para tratar ciertos temas específicos que ayudan al fortalecimiento organizacional de la misma. Por ello, el señor Luis Gomero nos menciona que hay una lucha constante, no solo para abrir mercados, sino, y más importante, para preservar los valles de Chillón, cuidarlos del uso de pesticidas y evitar que se cubran de cemento sus tierras, es decir, cuidarlas de las inmobiliarias. Por ello, una de las principales críticas que hace el presidente de la asociación a las instituciones municipales es que no tocan los problemas de fondo.

Sin embargo, han construido una relación estrecha con las municipalidades, y no solo con las aledañas sino también con las de diferentes distritos de Lima Metropolitana. Les invitan a actividades como ferias, talleres para evitar el uso de pesticidas y distintas conferencias de promoción de la agricultura saludable.

En el caso de los talleres, no solamente han sido impartidos por la municipalidad para las y los asociados, sino también han invitado abiertamente a personas interesadas en cambiar su modo de producir. Igualmente, podemos encontrar como evidencia las diferentes conferencias que han sido transmitidas en las plataformas de las redes sociales (Facebook y Youtube), en las que los mismos integrantes de la asociación fueron ponentes.

Pero está actividad (talleres de formación), también ha sido realizada por algunas ONG's, principalmente por Cuso International, que se define como una organización de desarrollo que trabaja para reducir la pobreza y la desigualdad, a través de los esfuerzos de voluntarios y voluntarias altamente calificados, asociaciones de colaboración y la generosidad de sus donantes. Esta organización les ha apoyado también con capacitaciones de producción y cuidado del medio ambiente. Los talleres han permitido que los asociados puedan seguir compartiendo puntos en común, así como seguir fomentando e invitando a unirse a la asociación. 
Otras de las actividades fundamentales donde las municipalidades ofrecieron su apoyo son las ferias agroecológicas, las cuales se desarrollaban habitualmente los domingos, es decir, una vez por semana. En ellas, la mayoría de los productores participa y promociona sus productos. Sin embargo, para algunos asociados no es suficiente un día a la semana, por lo que buscaron otras vías para la venta de sus productos como delivery y establecer puntos de ventas como el mercado Qatuna.

Los productores del Fundo Agroecológico HECOSAN, que es parte de la APEVCH, nos mencionaron que en el mercado Quntuna se llegó a abrir un punto orgánico, en el puesto 74, para poder vender sus productos saludables, lo cual permitió que algunas cosechas no se perdieran.

Por otro lado, los productores del Huerto Yanina, nos comentaron que por necesidad se tenía que cosechar hasta tres veces por semana. Por ello, incursionó en la implementación del servicio de delivery, primero a personas cercanas; pero ahora ha crecido y cuenta con más lugares de reparto, sobre todo por el nuevo contexto de pandemia Covid-19.

Por último, la APEVCH ha participado intercambiando experiencias y conocimientos con otras asociaciones. Es el caso de la visita por los huertos de los asociados de la Confederación Campesina del Perú en el 2019; sin embargo, estas relaciones no han sido muy duraderas, al igual que otras asociaciones.

\section{Escenarios a futuro}

Las metas de las y los productores integrantes de la APEVCH muestran su deseo de crecer. Por ello, una de sus metas a corto plazo es sumar nuevos miembros, tal como señala Gicella Igreda: «Se está juntando a más gente para pasar de los 30 integrantes fundadores a 40, y así para poder producir y comercializar más productos ecológicos» (G. Igreda, entrevista, 05 de septiembre de 2020).

Asimismo, desean fortalecer los vínculos existentes con la municipalidad de Carabayllo, la cual les ha facilitado la oportunidad de tener una bioferia semanal en el distrito, algo vital para ellas y ellos. Tal como señala Yanina Loayza: «Lo que se busca es mantener la bioferia de manera permanente para poder tener un mayor alcance al público consumidor, esto ya nos ayudaría a una mayor salida de nuestros productos que pueden perderse» (Y. Loayza, entrevista, 17 de septiembre de 2020).

Saben que, como organización joven, tienen sus potencialidades y sus propias limitaciones, ya que no es muy fácil trabajar organizadamente, puesto que las y los agricultores se encuentran acostumbrados a trabajar de manera individual. Ello lo han tomado como aprendizaje para poder fortalecer sus relaciones sociales y solidarias. Es por eso que tratan de mostrar que, organizadamente, se pueden obtener mejores ventajas comparativas, especialmente para colocar sus productos en el mercado, y que la idea de la producción y comercialización de productos orgánicos se puede extender a más agricultores, como lo señala Felipe Huallpa: «Tener el nombre de la asociación nos ha abierto muchas puertas ... antes tenía que contratar un taxi y llevar mi mercadería a la parada a venderlo al precio que sea para no perder, pero eso ha ido cambiando con el pasar de los años» (F. Huallpa, entrevista, 10 de septiembre de 2020).

\section{Reflexiones finales}

A partir de la experiencia con la APEVCH, ésta se puede describir como un ejemplo de economía social y solidaria, debido a que las relaciones que se han generado desde adentro de la asociación, son principalmente de solidaridad y reciprocidad, donde resalta la horizontalidad 
entre los miembros de la organización, quienes buscan sostener su compromiso como asociación. Esta dinámica también se halla en la idea de producción ecológica y de participación activa de los miembros, la cual persigue establecer una agenda y metas en común, frente a los problemas que deben enfrentar . Esto se corresponde con lo que Coraggio (2011) señalaba acerca de las economías que rompen con las relaciones de acumulación de capital, su eje central, el mismo que construye a un ser individualista y egoísta.

Con respecto a las relaciones de género dentro de la organización, se podría decir que hay una mayor participación femenina dentro de la asociación y que la división del trabajo no siempre está determinada por nociones de un rol específico y separado entre mujer y varón. Desde la lectura de Carrasco (2011), proponemos la implementación de un circuito de trabajo ampliado, en donde las labores domésticas y de cuidados para producir los bienes y servicios necesarios para la subsistencia y bienestar de los miembros del hogar sea considerado como un aspecto fundamental y necesario para el mantenimiento de la vida humana, ya que este permite la ampliación del ciclo de reproducción, que el sistema en términos monetarios-económicos no cubre netamente con el trabajo mercantil.

Desde que la APEVCH fue creada, no ha logrado constituir de manera regular la participación de los miembros; es decir, que conforme se ha ido desarrollando la asociación, algunos de sus miembros no han mantenido una participación constante, por lo que han entrado a una situación de inactividad. Sin embargo, los miembros activos muestran las condiciones fundamentales para poder considerarlos como una relación social y solidaria, dado que el trabajo que realizan es para todos y todas. Para la superación de esta dificultad sugerimos, desde la lectura de una ética cooperativista de Gibson-Graham (2011), que la transformación social y la educación pueden promover una reconstrucción económica y educar a todos los miembros, en especial a las generaciones más jóvenes, para fortalecer su compromiso con los principios cooperativos. Por ello, consideramos que si estos puntos se integran en la agenda de APEVCH, se podría generar una consciencia dentro de la asociación, articulando la idea de formar una nueva economía más justa, como pilar, y una práctica educativa constante que genere en los socios una economía ecológica.

Se puede concluir también que la asociación, si bien empezó con un grupo reducido de personas, han ido concientizando las prácticas de una agricultura saludable. Esa consigna de vida ha ido llamando la atención de los que ahora son asociados, así como la transparencia a la hora de desarrollar la agricultura, lo que ha hecho que los consumidores confíen más en sus productos, estimulando por ende la continuidad de la APEVCH.

La búsqueda de un mercado ha representado una férrea dificultad, la cual no se ha podido sanear hasta el momento desde que se creó la asociación. Esto genera una incertidumbre en los miembros de la asociación, quienes han empezado a buscar sus propios espacios para comerciar, y cuya consecuencia es la ruptura de la unidad que ha buscado transmitir la asociación. Para este punto vamos a volver a tomar como referencia a Gibson-Graham (2011), ya que como parte de las prácticas y la ética cooperativista enfatiza los principios de cooperación grupal y de universalidad, ya que estos puntos fortalecen la cooperación entre sus miembros y con los de otros movimientos cooperativistas. En contraste, la APEVCH no ha mantenido contacto con otras organizaciones, por lo que consideramos que podría servir que busquen relacionarse con otras asociaciones similares, de producción ecológica, para compartir y desarrollar experiencias que podrían facilitar nuevos espacios donde comerciar, y a su vez generar vínculos que servirían también para encontrar soluciones a sus problemas latentes y comunes, como la amenaza que representa la urbanización carente de planificación. 


\section{Bibliografía}

Aliaga, M. (2010). Situación ambiental del recurso hídrico en la cuenca baja del río Chillón y su factibilidad de recuperación para el desarrollo sostenible. Lima: Universidad Nacional de Ingeniería.

Andrade, C. (2017). Análisis sustentable de las fincas de brócoli en Santa Rosa de Quives - Lima. Lima: Universidad Nacional Agraria La Molina.

Cáceres, J. (2013). Cambios de Uso de Suelo y Políticas Públicas en la Cuenca Baja del Río Chillón - Sector de San Pedro de Carabayllo. Lima: Pontificia Universidad Católica del Perú.

Carrasco, C. (2011). La economía del cuidado: Planteamiento actual y desafíos pendientes. Revista de Economía Crítica, (11), 205-225.

Comisión Multisectorial “Año Internacional de la Agricultura Familiar-AIAF 2014”. (2015). Estrategia Nacional De Agricultura Familiar 2015 - 2021. Lima: Ministerio de Agricultura y Riego.

Comité de Agricultura (1999). La Agricultura Urbana Y Periurbana. Roma: FAO.

Coraggio, J. L. (2011). Economía Social y Solidaria. El trabajo antes que el capital. Quito: Ediciones Abya-Yala.

Cuentas, M.; Caballero, R.; Urday, P. y Santur, L. (2010). Inserción ventajosa de pequeños productores a mercados dinámicos: Cadenas de papa capiro y papa nativa. Lima: FOVIDA.

Diaz, G. (2019). Evaluación de residuos de plaguicidas en cultivos hortícolas en el valle Chillón. Huacho: Universidad Nacional José Faustino Sánchez Carrión.

FAO (2014). Agricultura familiar en América Latina y El Caribe: Recomendaciones de Política. Santiago de Chile: FAO.

Gibson-Graham, J. K. (2011). Una política poscapitalista. Medellín: Siglo del Hombre Editores.

Marañón, P. (2015). Manejo y uso de los plaguicidas agrícolas entre los horticultores en el valle del río ChillónLima. Lima: Universidad Nacional Agraria La Molina.

Mondragón, E. (2019). Conflictos ambientales por el uso de los recursos hídricos en la cuenca del río ChillónPerú. Lima: Universidad Nacional Federico Villarreal.

Presentado: 12/10/2020

Aceptado: 20/03/2021

Publicado online: 10/08/2021 